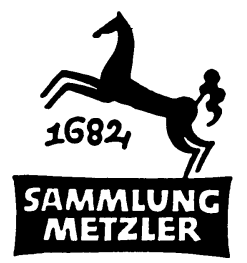

REALIEN ZUR LITERATUR

ABT. D:

LITERATURGESCHICHTE 
GERHARD P. KNAPP

\section{Georg Büchner}

2., neu bearbeitete Auflage

MCMLXXXIV

J. B. METZLERSCHE VERLAGSBUCHHANDLUNG

STUTTGART 


\section{Für Julia, Cordelia, Elaine und Hanna}

Le mot de Vertu vient de force, la force est la base de toute Vertu.

(Les Pensées de J. J. Rousseau, Citoyen de Genève)

CIP-Kurztitelaufnahme der Deutschen Bibliothek

Knapp, Gerhard P.:

Georg Büchner / Gerhard P. Knapp. -

2., neu bearb. Aufl., (6-10. Tsd.). -

Stuttgart : Metzler, 1984.

(Sammlung Metzler; M 159 : Abt. D, Literaturgeschichte)

ISBN 978-3-476-12159-2

ISBN 978-3-476-04091-6 (eBook)

DOI 10.1007/978-3-476-04091-6

NE: GT

\section{159}

(C) 1984 Springer-Verlag GmbH Deutschland

Ursprünglich erschienen bei J. B. Metzlersche Verlagsbuchhandlung und Carl Ernst Poeschel Verlag GmbH in Stuttgart 1977/1984 
I. Der Autor . . . . . . . . . . . . . . . . .

1. Herkunft und Familie $1-2$. Gymnasialzeit und Jugendschriften 5 - 3. Straßburg $11-4$. Gießen. Der Revolutionär 15 -5. Exil. Straßburg und Zürich 21-6. Der Naturwissenschaftler $26-7$. Nachwirkung 31

II. Der Hessische Landbote. Die politischen Anschauungen . . . . . . . . . . . . . .

1. Der Text 34 - 2. Die politischen Anschauungen 41 Literatur 45

III. Dantons Tod . . . . . . . . . . . . . . . . .

1. Entstehung und Textlage $47-2$. Zur Quellenlage $49-3$. Büchners Ästhetik der Montage 50 - 4. Struktur und Sprache 54 - 5. Geschichte als Leidensprozeß und Glücksverheißung 57 - 6. Die Positionen des Dramas 62 - a) Das Schöne 65-b) Das Notwendige $67-c$ ) Die letzten Dinge 73-7. Deutungsperspektiven 75 - Anhang: Die innerliterarische Wirkung; Vertonungen 78 - Literatur 80

IV. Lenz . . . . . . . . . . . . . . . .

1. Der Stoff und die Quellen 85-2. Die Form der Erzählung 89-3. Die Hauptaspekte der Erzählung 90-a) Das "Kunstgespräch « 91 - b) Der Glaubensverlust 94 - c) Die Krankheit 97 4. Deutungsperspektiven 99-Anhang: Schneiders Erzählung und Rihms Kammeroper 101 - Literatur 101

V. Leonce und Lena . . . . . . . . . . . . . . . . .

1. Datierung 103 - 2. Die Sprache 104 - 3. Struktur und Quellen 105 - 4. Kunstform als Kritik 107 - 5. Satirische Widerhaken $112-6$. Deutungsperspektiven 115-Anhang: die Vertonungen 118 - Literatur 118

VI. Woyzeck. . . . . . . . . . . . . . .

1. Überlieferung 120-2. Entstehung 122-3. Der Stoff und die Quellen 123 - 4. Die Sprache 125 - 5. Komposition 126-6. Lese- und Bühnentext 128 - 7. Krankheit und Gesellschaft 130

a) Die Problematisierung des Stoffes in den Entwürfen 130-b) Konturen des Sozialdramas $136-8$. Deutungsperspektiven 138 - Anhang: Csokors »Woyzeck «-Vollendung; Bergs "Wozzeck" 140 - Literatur 141 
VII. Anmerkungen zur Forschungslage . . . . . . . . . 144

VIII. Auswahlbibliographie . . . . . . . . . . . 150

Register . . . . . . . . . . . . . . . 169 
AUMLA Journal of the Australasian Modern Language Association

B

DRds

DU

DVJs

EG

Euph

GAG

GLL

GN

GQ

GR

GRM

$\mathrm{H}$

JbIG

JEGP

MLR

Monatshefte

$\mathrm{NdL}$

NGS

NRS

OGS

OL

PMLA

RLV

Seminar

SM

SuF

TDR

WB

WW

$\mathrm{ZfdPh}$

ZfG

ZsfDkd
Georg Büchner. Werke und Briefe. Gesamtausgabe hg.v. Fritz Bergemann. Wiesbaden ${ }^{8} 1958$.

Deutsche Rundschau

Der Deutschunterricht

Deutsche Vierteljahrsschrift für Literaturwissenschaft und Geistesgeschichte

Etudes Germaniques

Euphorion. Zeitschrift für Literaturgeschichte

Göppinger Arbeiten zur Germanistik

German Life and Letters

Germanic Notes

The German Quarterly

The Germanic Review

Germanisch-Romanische Monatsschrift

Georg Büchner. Sämtliche Werke und Briefe. Historisch-

kritische Ausgabe mit Kommentar, Hg.v. Werner $R$.

Lebmann $=$ Hamburger Ausgabe. Bislang erschienen

Bd. I Hamburg 1967 [zit. als H1] und Bd. II Hamburg 1971 [zit. als H2].

Jahrbuch für Internationale Germanistik

Journal of English and Germanic Philology

The Modern Language Review

Monatshefte für deutschen Unterricht, deutsche Sprache und Literatur

Neue deutsche Literatur

New German Studies

Neue Rundschau

Oxford German Studies

Orbis Litterarum

Publications of the Modern Language Association of America

Revue des langues vivantes

Seminar. A Journal of Germanic Studies

Schweizer Monatshefte für Politik, Wirtschaft und Kultur

Sinn und Form

Tulane Drama Review

Weimarer Beiträge

Wirkendes Wort

Zeitschrift für deutsche Philologie

Zeitschrift für Germanistik (Korea)

Zeitschrift für Deutschkunde 
Die Büchnerforschung hat im vergangenen Jahrzehnt eine Vielzahl neuer Ergebnisse verbuchen können. Bestehende Kontroversen haben sich dabei vielfach verschärft, die Fronten um eine sachgerechte Beurteilung von Autor und Werk verhärten sich zusehends. Allein im Hinblick auf den vermehrten Wissensstand von heute erschien eine einfache Überarbeitung der ersten Auflage dieses Bandes als nicht hinreichend. Nur das erste Kapitel bleibt, allerdings in deutlich vermehrtem Umfang, erhalten. Alle anderen Teile des Buchs wurden auf der Grundlage der wichtigsten neueren Forschungsergebnisse neu erarbeitet. Auch diesmal sind wieder eine Reihe eigener Resultate - die durchweg über die Konzeption der Erstauflage hinausführen - in die Darstellung eingeflossen. Im Sinne einer besseren Benutzbarkeit wurde in der vorliegenden Neuauflage das ursprüngliche Netz von Verweisen auf die Sekundärliteratur beträchtlich erweitert. Von hier aus dürfte sich dem Leser das beachtliche wissenschaftliche Umfeld von Tatsachen, Thesen und Versuchen relativ mühelos erschließen.

Danken möchte ich an dieser Stelle meiner Frau, die die Arbeit an diesem Band mit Rat, Unterstützung und gutem Mut begleitet hat. Auch die Institutionen, Bibliotheken und Institute, die das Projekt auf vielfältige Weise über eine Reihe von Jahren förderten, verdienen meinen Dank. Den Kollegen, die durch Kritik und praktische Hilfe sowie durch die freundliche Überlassung von Handschriftenkopien das Zustandekommen der Studie ermöglichten, bin ich zu Dank verpflichtet. 